\title{
VISUALISIERUNG UND IHRE FUNKTIONEN BEI DER NOTIZENNAHME IM KSD ${ }^{1}$
}

\author{
LUCYNA BRZOZOWSKA \\ Adam Mickiewicz University - Poznań
}

\begin{abstract}
Taking notes in consecutive interpreting is of eminent significance. It is directly related to the specificity of consecutive interpreting, in the first line to the phase displacing: at first there is a text presented to be translated and afterwards its respective translation is produced. Written texts which are read aloud are more difficult in the reception than spoken texts. Even more difficult are specialized texts. If a specialized written text is presented orally in longer sequences the consecutive interpreter is able to translate it in the proper way only with help of taking notes.
\end{abstract}

Die Notizennahme ist beim KSD von eminenter Bedeutung. Ihr Ziel ist die Entlastung des Translators bei Speicherung und Erinnerung. Mit Hilfe von Notizen werden nicht einzelne Wörter, sondern ganze Sinneinheiten auf dem Papier fixiert ${ }^{2}$, was dem Translator als Grundlage für die Memorierung des Rezipierten dient. Die Notation hängt dadurch eng mit der Spezifik des KSD-Vorganges zusammen.

\section{Die Spezifik des KSD}

Das $\mathrm{KSD}^{3}$ ist eine Realisierungsvariante des Translationsprozesses. Es wird von anderen Translationsarten anhand verschiedener Kriterien unterschieden, die sich auf die Ausführung der Darbietung des AS-Textes und dessen Rezeption

\footnotetext{
' Abkürzungsverzeichnis: AS - Ausgangssprache, KSD - Konsekutivdolmetschen, ZS - Zielsprache.

${ }^{2}$ Heinz Matyssek (1989:164) schlieBt dabei die Stenographie aus, denn sie zielt darauf ab, Aussagen zwecks ihres Wiederlesens wortgetreu zu fixieren.

${ }^{3}$ In der einschlägigen Literatur sind verschiedene Definitionen des KSD vorhanden, in Abhängigkeit davon, welche Aspekte der jeweilige Autor bei der Explikation für relevant hält. In dem vorliegenden Beitrag wird das KSD im Sinne von Zmudzki (1995) verstanden. Zmudzki definiert das KSD (1995:41) als eine Sondersorte der Translation, die als „Handlung nach der AS-sequentiellen mündlichen Vorgabe eines mùndlich verfertigten AS-Textes (als Handlung) postsequentiell und mündlich in einer ZS vollzogen wird", und seine Spezifik beruht nicht darauf, daß es durch die jeweiligen Vollzugsbedingungen ,zu einer besonderen Translationsart wird, sondern da $\beta$ es einen besonderen Handlungstransfer realisiert und dadurch die Funktion einer transferierenden Handlung bekommt".
} 
sowie auf inhärente Merkmale einzelner mentaler Operationen beziehen. Eine Auflistung der wichtigsten Klassifikationskriterien bei der Explikation des KSD, die in der KSD-Fachliteratur genannt werden, nimmt Żmudzki (1995:19) vor. Es sind:

- die Phasenverschiebung d.h. die Aufeinanderfolge der Rezeption des AS-Textes und der Produktion des ZS-Textes;

- die Mündlichkeit, aus der die Einmaligkeit des phonetisch dargebotenen AS-Textes und die Form der ZS-Textverfertigung resultiert;

- die auf die Darbietungszeit beschränkte Existenzdauer des AS-Textes, die aus der Einmaligkeit resultiert;

- die Auditivität der Rezeption;

- die auf die Rezeptionsdauer begrenzte Umkodierungszeit.

Konstitutiv für das KSD ist die Phasenverschiebung: Zuerst erfolgt die Aufnahme des AS-Textes und erst danach die Produktion eines entsprechenden ZSTextes.

Aus dem obigen ergibt sich die Einteilung des KSD-Vorgangs in Teilhandlungen, wobei sich, trotz aller Vielfalt der Gesichtspunkte, folgende Hauptphasen ${ }^{4}$ unterscheiden lassen (Żmudzki 1995:20-24):

- die Phase der Rezeption;

- die Phase der Transposition;

- die Phase der Realisation.

Ein komplexes Modell des KSD stellt Jerzy Źmudzki (1995) dar. Es besteht aus folgenden Phasen:

- Initialphase;

- Vollzug der AS-Texthandlung;

- Rezeption des AS-Textes vom Translator;

- Projektion des AS-Textes auf die ZS und die ZS-Kultur;

- Konstruktion der ZS-Texthandlungen;

- sprachlicher Vollzug der ZS-Texthandlung;

- Monitoring, Kontrolle und Steuerung als Aktivitätsbereiche des ControllingSystems.

Jeder Vollzug des KSD ist somit ein mehrdimensionaler Akt, dem bestimmte Handlungen, Operationen und Strategien zugrunde liegen, die über seine Spezifik entscheiden. Die Entscheidung über konkrete, für einen jeweiligen KSD-

${ }^{4}$ Es muß dabei darauf hingewiesen werden, daß in der Fachliteratur verschiedene Termini für die Bezeichnung der KSD-Phasen gebraucht werden (Kade 1963; Matyssek 1989; Willet 1974; Zmudzki 1995). 
Vollzug geeignete Handlungen, Operationen und Strategien trifft der Translator/Dolmetscher in der Initialphase in Abhängigkeit von den jeweiligen Vollzugsbedingungen.

Berücksichtigt wird dabei die gesamte Umgebungssituation. Daraus resultieren folgende Fragestellungen: Welches Thema betrifft der AS-Text? Wer ist der Translationsinitiator? Wer ist der AS-Textproduzent? Ist es dieselbe Person? Welche Merkmale hat der AS-Text? Ist es ein geschriebener Text, der nur vorgelesen wird oder ein gesprochener? Ist es ein Fachtext oder ein allgemeiner Text? Verfügt der Translator im Falle eines Fachtextes über das entsprechende Fachwissen? Wie wurde die Translationsaufgabe formuliert? Wie ist der Handlungsrahmen? Wie ist die soziale Relation zwischen Translationspartizipanten? Ist die Notizennahme möglich bzw. notwendig und überhaupt angebracht ${ }^{5}$ in der jeweiligen Translationssituation als Kommunikationssituation?

Die Antwort auf die letzt gestellte Frage hängt vor allem von der Translationsaufgabe und von dem zu dolmetschenden Text ab.

Die Translationsaufgabe spielt eine entscheidende Rolle für die Auswahl der Transferart. Zmudzki (1995:46) unterscheidet 3 Grundtypen des Transfers:

- den direkten, simulierenden Handlungstransfer, im Rahmen dessen eine in der AS vollzogene Texthandlung funktional und referentiell vom Translator erfaßt/simuliert wird; es wird ausgewählt, wenn der Translator laut der Translationsaufgabe eine ASTexthandlung in der ZS simulieren soll;

- den deskriptiven Handlungstransfer, im Rahmen dessen eine in der AS-Texthandlung ausgedrückte Intention vom Translator hinsichtlich der zu vollziehenden ZSTextillokution mit der ZS-Textreferenz umgewandelt wird; in diesem Fall nimmt der Translator gemäß der Translationsaufgabe eine ZS-Deskription der AS-Texthandlung vor;

- den synthetisierenden Handlungstransfer, im Rahmen dessen die AS-Texthandlung vom Translator in ihre deskriptive Synthese umgewandelt wird; es wird ausgewählt, wenn die Translationsaufgabe dem Translator eine ZS-Synthese der AS-Texthandlung vorgibt.

Fernerhin hängt die Auswahl des jeweiligen Handlungstransfers von der ASTextart ab, denn geschriebene und gesprochene Texte sowie allgemein- und fachsprachliche Texte unterscheiden sich wesentlich voneinander und erfordern somit entsprechende Translationsstrategien und Transfertypen.

Die Translationsaufgabe, die Textart und der Transfertyp entscheiden darüber, ob die Notation notwendig und überhaupt angebracht ist, und fernerhin beeinflussen sie auch die Art der Visualisierung bei der Notizennahme.

\footnotetext{
${ }^{5}$ Die Notizennahme ist in manchen Translationssituationen nicht angebracht, z.B. bei offiziellen Empfängen, feierlichen Ansprachen.
} 


\section{Gesprochene und geschriebene Texte, Fachtexte und allgemeinsprachliche Texte}

Geschriebene Texte, die vorgelesen werden, sind wesentlich schwieriger in der Rezeption als gesprochene. Geschriebene Texte sind durch eine komplizierte Syntax, lange Satzperioden und die Anhäufung der Begriffe gekennzeichnet. Noch schwieriger sind Fachtexte ${ }^{6}$ mit all ihren spezifischen Sprachmitteln, etwa (Hoffmann 1987:230, 239):

- die große Häufigkeit von Simplizia, Derivativa, Lehnwörtern, Internationalismen und vor allem Fachwörtern und Termini;

- häufiger Gebrauch von Nominalphrasen und Verbalphrasen;

- ausgiebiger Gebrauch von Symbolen, Zahlangaben, Begriffsauflistungen und Formeln;

- Besonderheiten in der Anordnung der Satzglieder, wie z.B. die Frontstellung präpositionaler Objekte und ganzer Objektgruppen oder die Initialposition des Prädikats;

- große Häufigkeit anaphorischer Elemente am Satzanfang;

- große Anzahl von einfachen erweiterten Sätzen und Satzgefügen mit untergeordneten Nebensätzen; Dominanz von konditionalen Nebensätzen;

- Überwiegen der funktionalen Satzperspektive;

- bei Verben: Gebrauch von Indikativ, Präsens, 3. Person, Passiv; bei Substantiven: Singularform und Genitivfall;

- Wortwiederholungen statt (anaphorischer) Pronominalisierung und Subordination; direkte thematische Progression;

- Typische Makrostruktur: in der Überschrift wird zuerst der Gegenstand genannt und dem folgen Problemstellung, Prämissen, Methode, Lösung, Anwendung.

$\mathrm{Zu}$ den allgemeinen satzgrenzenüberschreitenden Merkmalen eines Fachtextes zählen (nach Golovin, Hoffmann 1987:231) die Gebundenheit, Präzisiertheit und Gegliedertheit der Rede im sog. wissenschaftlichen Stil.

Die Besonderheit der Fachtexte ergibt sich zuletzt daraus, daß sie sich mit einem bestimmten Fachgebiet befassen. Ein Fachtext (Hoffmann 1987:233) wird von einem Fachmann für einen Fachmann (seltener einen Nicht-Fachmann) erzeugt, der dem rezipierten Text mit einer bestimmten Erwartungshaltung entgegentritt. Den Bezugsrahmen für beide Kommunikationspartner bildet dabei der jeweilige Fachbereich, in dem sowohl der Textproduzent als auch der Textrezipient bewandert

'Ein Fachtext wird in dem vorliegenden Beitrag nach der Definition von Hoffmann (1987:234-235) wie folgt verstanden als ,ein Instrument und Resultat der im Zusammenhang mir einer spezialisierten gesellschaftlich-produktiven Tätigkeit ausgeübten sprachlich-kommunikativen Tätigkeit; er besteht aus einer endlichen, geordneten Menge logisch, semantisch und syntaktisch kohärenter Sätze (Texteme) oder satzwertiger Einheiten, die als komplexe sprachliche Zeichen komplexen Propositionen im Bewußtsein des Menschen und komplexen Sachverhalten in der objektiven Realität entsprechen". 
sind. Der Translator muß dieser Erwartungshaltung und somit den Anforderungen an das notwendige Fachwissen nachkommen, um die Translationsaufgabe erfolgreich zu bewältigen.

\section{Die Notwendigkeit der Notizennahme}

Wenn ein geschriebener AS-(Fach)text mündlich in einer Translationssituation in längeren Sequenzen dargeboten wird und der Translator im Rahmen der Translationsaufgabe eine Simulierung oder eine Deskription der AS-Texthandlung in der ZS vornehmen soll (und umso mehr wenn er über das entsprechende (Fach)wissen nicht verfügt), wird seine Gedächtniskapazität ${ }^{7}$ in so hohem Grade belastet, daß er kaum imstande ist, der Translationsaufgabe gerecht zu werden, d.h. die Gedanken und Absichten des AS-Textproduzenten sinngenau, sprachrichtig, stiladäquat und wirkungsgleich an den ZS-Textrezipienten heranzutragen. In solchen Fällen scheint die Notizennahme unentbehrlich. Es geht dabei nicht um das bloße lineare Mitschreiben der gehörten Wörter, sondern um die schriftliche Fixierung des rezipierten Inhalts in der einfachsten Form als Aktivierung, Gedächtnisstütze und Ergänzung der Speicherung. Im Falle einer ZS-Synthese der AS-Texthandlung ist die Notizennahme nicht notwendig, da der Translator eine gezielte Selektion der für die Translationsaufgabe relevanten AS-Textteile vornimmt und seine Gedächtniskapazität nicht in so einem Maße beansprucht wird wie beim simulierenden oder deskriptiven Transfer.

Ein guter Translator mit einem geschulten ,Dolmetschergedächtnis“ und einer guten Kenntnis des jeweiligen Themenbereiches soll (Matyssek 1989:25) ca. 60-80\% des AS-Textes im kognitiven Gedächtnis speichern und den Rest in Form von Notizen schriftlich fixieren. Bei geringer Sach- und Situationsvertrautheit muß sich der Translator in Abhängigkeit von der Translationsaufgabe in einem noch höherem Grade auf die Notation verlassen, um den in seinem Gedächtnis gespeicherten Inhalt zu ergänzen. Für einige Autoren ist die Notation so wichtig, daß sie zu den Klassifikationskriterien des KSD gezählt und damit zu den Bestandteilen des KSD-Vorgangs aufgenommen wird (Żmudzki 1995:25).

Auf die Notwendigkeit der materiellen Fixierung der Speicherungsdaten beim KSD weisen u.a. Matyssek (1989), Kade (1963), Willet (1974) und Kalina (1998) hin. Kalina (1998:21) versteht das KSD folgendermaßen:

Beim Konsekutivdolmetschen hört der Dolmetscher entweder einen vollständigen Diskurs in der AS und gibt ihn wieder, oder es werden Teilstücke des Diskurses von einer Länge zwischen ca. drei und zwölf Minuten vorgetragen, wobei vom

${ }^{7}$ Es handelt sich dabei um das Kurzzeitgedächtnis. In dem vorliegenden Beitrag wird jedoch darauf nicht näher eingegangen, denn es würde seinen Rahmen sprengen (mehr dazu in Kurcz 1992; Cartellieri 1979). 
Redner jeweils Pausen für die Dolmetschung eingelegt werden. Die zielsprachliche Wiedergabe kann aus dem Gedächtnis erfolgen; Da die Gedächtniskapazität des Dolmetschers nicht unendlich ist, macht er sich im allgemeinen Notizen, die ihm die Memorierung des Gehörten erleichtern.

Einer ähnlichen Ansicht ist Willet (1974:99). Der Translator hört/versteht und assimiliert den AS-Text und anschließend reproduziert er die assimilierten Gedanken aus dem Gedächtnis oder auch mit Hilfe von Notizen. Die Notizen übernehmen bei Willet die Funktion einer Erinnerungshilfe, die einen schnellen Abrufungsprozeß und einen leichteren Output ermöglichen. Die Notwendigkeit der Notizennahme hebt auch Kade (1963:14) hervor, denn die Notizen unterstützen die Speicherung des relevanten ${ }^{8}$ begrifflichen Inhalts des AS-Textes.

Einer noch restriktiveren Auffassung ist Heinz Matyssek (1989:22), für den die Phasenverschiebung beim KSD kein Abgrenzungskriterium gegenüber dem Simultandolmetschen ist, sondern es sind die Vollzugssituation (eine Konferenz ${ }^{9}$ ) und der in Form von Notizen schriftlich gespeicherte Aussagekern des AS-Textes:

Diese Speicherung vollzieht sich in zwei Formen bzw. auf zwei Wegen:

$\checkmark$ in Form der (kognitiven, nicht mechanischen $=$ nicht an der Worthülse orientierten!) Speicherung des durch die „Assimilation“ = geistige Aneignung also Hinübernahme des Redeinhalts aus der Sphäre des Verstehens in die Sphäre des wissensverfestigten Sinngehalts - im Gedächtnis.

$\checkmark$ in Form der „materiell" fixierenden Speicherung des geistigen Aussagegerüsts, des Aussagekerns durch die Notation auf dem Block.

Die beste Form dafür bietet ein Notationssystem, mit dessen Hilfe der Dolmetscher relevante Informationen auf dem Block verankern kann. Die Notizennahme hat beim KSD somit 2 Funktionen:

- die Steigerung der Speicherung - dank der Notizen verläuft sie effektiver;

- die Ergänzung der Speicherung - der Translator fixiert schriftlich in Form von Notizen die Einzelheiten des AS-Textes, die er nicht imstande ist, in seinem Gedächtnis zu speichern.

\section{Die Matyssek'sche Notizentechnik}

In der Fachliteratur sind mehrere Notiersysteme vorgeschlagen worden: von Herbert (1952), von Rozan (1956), von Minjar-Belorutch'ev (1969) und von

\footnotetext{
${ }^{8}$ Nach Kade (1963:14) beruht die Verarbeitung des begrifflichen Inhalts des AS-Textes darauf, daß die semantisch relevanten sprachlichen Einheiten von den redundanten getrennt werden. Der Translator geht also selektiv bei der Verarbeitung vor.

${ }^{9}$ Matyssek (1989:8) vertritt die Ansicht, daß das KSD (neben dem Simultandolmetschen) eine Variante des Konferenzdolmetschens ist.
} 
Matyssek (1989). Unter den drei genannten Systemen ist das Matyssek`sche am weitesten ausgearbeitet und eben ihm gilt das Interesse des vorliegenden Beitrags.

Heinz Matyssek hat jahrelange Erfahrungen als Dolmetscher gesammelt und anhand dieser seine Notizentechnik erarbeitet. Seiner Ansicht nach müssen Notizen, um als Gedächtnisstütze erfolgreich zu fungieren, bestimmte Anforderungen erfüllen. Effiziente Notizen (Matyssek 1989:48-50) sind somit:

- einfach;

- klar;

- ökonomisch;

- unverwechselbar;

- schnell erfassbar;

- bildhaft.

Das Notiersystem von Matyssek zielt darauf ab, den Translator von der sprachlichen Form zu befreien, was sich darin widerspiegelt, daß es sich neben den Abkürzungen vor allem aus Symbolen (,Nicht-Wort“-Symbolen) zusammensetzt. Mit der Anwendung von Symbolen (Matyssek 1989:38) können die Notizen weitestgehend „wortfrei“" oder „sprachlos“, d.h. „ohne Wortgestalt" angefertigt werden ${ }^{10}$.

Die Untersuchungen von Kalina (1998:182) zeigen, daß Symbole und individuelle Abkürzungen in den Notationen in starkem Maße Verwendung finden, wobei die Versuchspersonen im Laufe ihrer Ausbildung Unterweisungen in der Matyssek'schen Notizentechnik erhielten. Es heißt, daß das Notationssystem von Heinz Matyssek im Dolmetschalltag als eine effiziente Hilfe fungiert.

\section{Die Visualisierung bei der Notizennahme}

Bei der Notizennahme mit Hilfe der Matyssek'schen Notizentechnik entsteht ein graphisch-textueller Plan des AS-Textes, der dem Translator als Gedächtnisergänzung und Fixierungsmittel dient. Es geht dabei darum, daß die Topologie des AS-Textes erhalten bleibt. Der Translator nimmt durch die Anwendung von Symbolen und Abkürzungen die Symbolisierung dessen vor, was er als relevant für die Translationsaufgabe erkannt hat.

Es werden mehrere Arten der Symbolisierung unterschieden. Bei der Notizennahme im KSD geht es um eine textartige Visualisierung mit Hilfe von ikonischen und graphemischen Elementen:

\footnotetext{
${ }^{10}$ In der einschlägigen Literatur sind verschiedene Ansichten bezüglich der Sprache, in der notiert werden soll, zu finden. Heinz Matyssek (1989) befürwortet die Muttersprache als Notiersprache.
} 
- textartige, weil ganze Texte dem Transfer unterliegen und sich ein Text aus den Notizen ergeben muB; dabei geht es um zwei Transfervorgänge;

- ikonische, denn es werden ganze Sinneinheiten (Wörter, Phrasen und Sätze) in Form von Ikonen (Symbolen) fixiert;

- graphemische, weil gängige Abkürzungen neben den Ikonen verwendet werden, wie z.B. EU, WHO, FAZ, Uni.

Einer der Transfervorgänge beruht darauf, daß der Translator den AS-Text in der ZS unter Berücksichtigung der zielsprachlichen Spezifik, des zielsprachlichen Textrezipienten und der Spezifik des ZS-Textes umprofiliert. In dem Fall wird ein kognitiv-evokativer Transfer vorgenommen. Der Translator muß, um den AS-Text zu rezipieren, d.h. Reize aufnehmen und sie kognitiv verarbeiten zu können, muß sich der Welt gegenüber sensorisch öffnen.

Dem Transfer unterliegt auch die Verarbeitung des AS-Textes. In einer Translationssituation als Kommunikationssituation, in der notiert wird, wird eine materielle Form für die Repräsentationen von Ideen gebildet, und erst diese werden als Perzepte wahrgenommen und weiterverarbeitet. In dem Fall wird die Textform transferiert: ein mündlicher Text wird in einen ikonischen und graphemischen Text auf dem Block umgewandelt.

Die Auswahl der Visualisierungsart in einer Translationssituation steht im engen Zusammenhang mit der Translationsaufgabe, dem Transfertyp und der AS-Texthandlung. Im Falle der Fachtexte ist die graphemische Visualisierung adäquater, denn sie ermöglicht eine schnelle und exakte Fixierung von Termini, Fachwörtern, Formeln und Zahlenangaben. Die ikonische Visualisierung eignet sich dagegen besser für allgemeinsprachliche, bei denen die Exaktheit nicht so gefragt ist. Die Hauptfunktion der Visualisierung in der Notizennahme beim KSD besteht somit darin, daß der Translator mit Hilfe von Ikonen (Symbolen) und Graphemen (Abkürzungen) einen mündlichen AS-Text als Gedächtnissteigerung und Gedächtnisergänzung schriftlich fixiert, um sich mit den Notizen bei der Produktion des ZS-Textes zu behelfen und somit die Translationsaufgabe zu bewältigen.

\section{Fazit}

Das KSD ist eine Sondersorte der Translation, die sich von den anderen Translationssorten durch Merkmale wie die AS-sequentielle mündliche Vorgabe eines mündlich verfertigten AS-Textes (als Handlung) und der postsequentielle mündliche Vollzug eines entsprechenden ZS-Textes unterscheidet.

Die Notizennahme beim KSD hilft dabei, die Memorierung der relevanten AS-Texteinheiten zu steigern und zu ergänzen. Bei einigen Autoren (Matyssek 1989; Kade 1963; Willet 1974; Kalina 1998) findet sie sogar Niederschlag in der Unterscheidung der KSD-Phasen. 
Bei der Notizennahme geht es nicht um das wortgetreue Mitschreiben des AS-Textes, sondern um die materielle Fixierung der relevanten Sinneinheiten, was eine Stütze für die kognitive Speicherung im Gedächtnis bildet. Es wird dabei ein besonderer Transfertyp vollzogen - die Informationen, die der Konsekutivdolmetscher für relevant hält, werden visualisiert, d.h. in Form von Ikonen und Graphemen dargestellt. Die Notizennahme und die Art der Visualisierung hängen von der Translationsaufgabe, dem Transfertyp und der AS-Texthandlung ab. Dank der Visualisierung löst sich der Translator vom Wortgerüst des AS-Textes und wendet sich dem Textinhalt zu, der durch die Anwendung von Ikonen und Graphemen mit einem Blick erfassbar wird.

\section{LITERATUR}

Cartellieri, C. (1979): Zu Bedeutung und Aufgaben des Gedächtnisses beim Dolmetschen. In: Fremdsprachen 23/3, 169-178.

Herbert, J. (1952): The Interpreter's Handbook. Geneve.

Hoffmann, L. (1987): Kommunikationsmittel Fachsprache. Eine Einfiihrung. Berlin.

Kade, O. (1963): Der Dolmetschvorgang und die Notation - Bedeutung und Aufgaben der Notiertechnik und des Notiersystems beim konsekutiven Dolmetschen. In: Fremdsprachen I, 12-20.

Kade, O. (1980): Die Sprachmittlung als gesellschaftliche Erscheinung und Gegenstand wissenschaftlicher Untersuchung. Leipzig.

Kalina, S. (1998): Strategische Prozesse beim Dolmetschen. Tübingen.

Kielar, B. (1988): Ttumaczenie i koncepcje translatoryczne. Warszawa - Wrocław.

Kurcz, I. (1992): Pamięć. Uczenie się, Język. Warszawa.

Matyssek, H. (1989): Handbuch der Notizentechnik für Dolmetscher. Ein Weg zur sprachunabhängigen Notation. Heidelberg.

Minjar-Belorutch'ev (1969): Posobye po ustnomu perevodu. Moskwa.

Reiß, K.; Vermeer, H.J. (1991): Grundlegung einer allgemeinen Translationstheorie. Tübingen.

Rozan, J.- F. (1956): La prise de notes en interpretation consecutive. Genf.

Salevsky, H. (1986): Probleme des Simultandolmetschens. Eine Studie zur Handlungsspezifik (Linguistische Studien Reihe A 154). Berlin.

Willet, R. (1974): Die Ausbildung zum Konferenzdolmetscher. In: Kapp, V. (1991): Übersetzer und Dolmetscher. Tübingen, 87-109.

Żmudzki, J. (1995): Konsekutivdolmetschen. Handlungen - Operationen - Strategien. Lublin. 